\title{
IMAGING TECHNIQUES FOR THE EVALUATION OF GRAPES IN WITHERING FOR AMARONE WINE PRODUCTION
}

\author{
BARBARA CISTERNA (*), FEDERICO BOSCHI (**), \\ ANNA CLETA CROCE (***), RACHELE PODDA (*), \\ SERENA ZANZONI (****), DANIELE DEGL'INNOCENTI (*), \\ PAOLO BERNARDI (*), MANUELA COSTANZO (*), \\ PASQUINA MARZOLA $(* *)$, VIVIANA COVI $(* * * * *)$, \\ GABRIELE TABARACCI $(* * * * *)$, MANUELA MALATESTA $(*)$
}

\begin{abstract}
SuNTO. - L'Optical Imaging (OI) è una tecnica sviluppata, in anni recenti, come approccio versatile, veloce e non invasivo per l'acquisizione di immagini di piccoli (pochi centimetri) campioni, come film di cellule (in vitro), piccoli animali (in vivo), organi di animali (ex vivo) e materiali innovativi. L'OI ha principalmente un' applicazione biomedica per lo studio di alcune patologie e per valutare l'efficacia di nuovi composti farmaceutici. Noi qui abbiamo applicato l'OI a un campo completamente nuovo: lo studio delle proprietà del cibo. Abbiamo, infatti, sfruttato le proprietà ottiche di molecole endogene, che generalmente sono considerate responsabili di un rumore di fondo che disturba l'acquisizione. Usando questo "rumore", chiamato autofluorescenza, abbiamo ricavato dati sull'appassimento delle uve di Corvinone impiegate per la produzione del vino Amarone. L'OI può, quindi, fornire interessanti informazioni e, in un approccio multimodale, potrebbe rappresentare un efficace supporto ad altre tecniche per descrivere un fenomeno biologico.
\end{abstract}

$* * *$

ABSTRACT. - Optical Imaging (OI) is an emerging field developed in recent years which can be a very versatile, fast and non-invasive approach for the acquisition of images of

(*) Dipartimento di Neuroscienze, Biomedicina e Movimento. Università degli Studi di Verona, Italia. E-mail: barbara.cisterna@univr.it

${ }^{(* *)}$ Dipartimento di Informatica, Università degli Studi di Verona, Italia.

${ }^{(* * *)}$ Istituto di Genetica Molecolare del CNR, Pavia, Italia.

$(* * * *)$ Centro Piattaforme Tecnologiche, Università degli Studi di Verona, Italia.

$(* * * * *)$ Poliambulatorio San Rocco, Montichiari (BS), Italia. 
small (few centimetres) sized samples, such as layers of cells (in vitro), small animals (in vivo), animal organs (ex vivo) and innovative materials. OI was primarily developed for biomedical applications to study the progression of some pathologies and to assess the efficacy of new pharmaceutical compounds. Here we applied the OI technique to a completely new field: the study of food optical properties. In this case we exploited the optical properties of endogenous molecules, which are generally considered responsible of a background noise affecting the investigation. Here we used this sort of "noise", named autofluorescence, to obtain information on the drying of Corvinone grapes employed for Amarone wine production. OI can provide interesting information and, inserted in a multimodal approach, it may be a real support to other techniques in the description of a biological phenomenon.

\section{INTRODUCTION}

Optical imaging (OI) is an emerging field developed in recent years, which can be a very versatile, fast and non-invasive (like photography) approach for the acquisition of images of small (few centimetres) sized samples, such as layers of cells (in vitro), small animals (in vivo), animal organs (ex vivo) and innovative materials. It is based on the detection of optical photons in the ultraviolet (UV, $10-400 \mathrm{~nm}$ ) and visible (VIS, $400-700 \mathrm{~nm}$ ) ranges, and in near infrared regions (NIR, $700 \mathrm{~nm}-1 \mathrm{~mm}$ ) of the electromagnetic spectrum. Thanks to the recent development of very sensitive detectors (photomultipliers and charge coupled devices - CCDs), it is possible to reveal very faint light signal in biological samples, in particular in the NIR region of the light spectrum. It is worth noting that only NIR photons are capable of travelling with minimal absorption throughout the biological tissues. NIR photons are thus able to reach deeper levels in the tissues.

OI was developed principally for biomedical applications to help other imaging techniques (such as magnetic resonance imaging, positron emission tomography, ultrasound echography) in the study of many pathologies and to assess the efficacy of new pharmaceutical compounds. Moreover OI, when applied in a multimodal imaging approach, allows to obtain information which can be compared and correlated with the results acquired with techniques such as microscopy.

OI is able to reveal light sources inside a biological sample. Generally, as we will see below, exogenous sources (e.g. fluorescent dyes or light-producing engineered cells) are employed. Here we applied OI technique to a completely new field: the study of food properties. 
In this case we exploited the optical properties of endogenous molecules, which are generally considered responsible of a background noise related to internal substances, which affect the investigation. Here we used this sort of "noise", named autofluorescence, to obtain information on the drying of Corvinone grapes employed for Amarone wine production. An example of fluorescence due to endogenous fluorescent molecules in fruits is shown in Fig. 1. The images, in pseudo-colours related to the intensity of the light emission, reveal the distribution of fluorescent molecule in tangerine. The investigation of which molecule(s) are responsible for the fluorescence emission is a very difficult task.

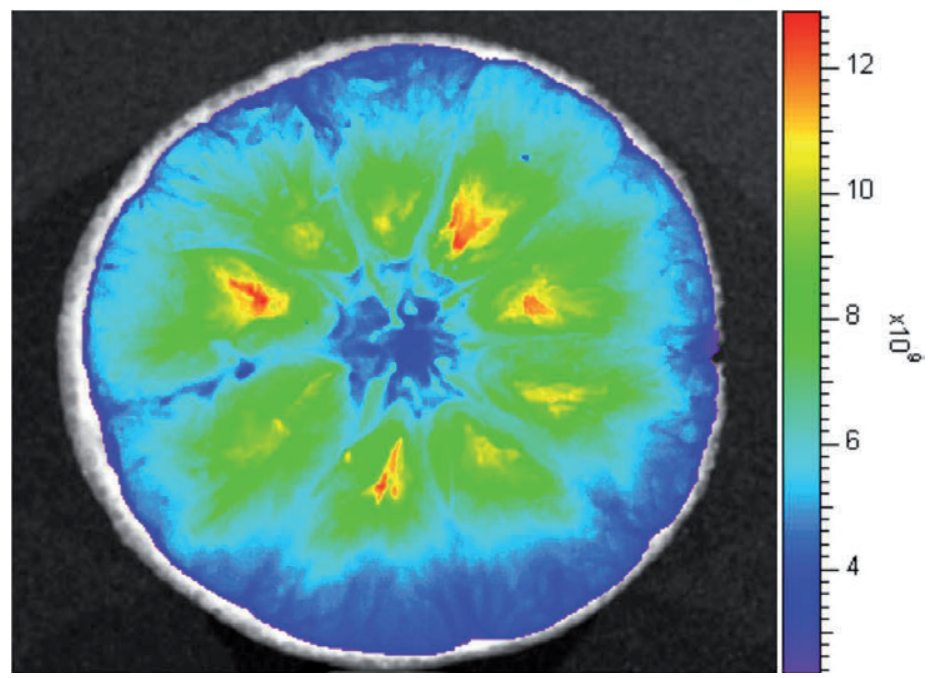

Fig. 1. Fluorescence image of tangerine obtained with 580-610 excitation filter and 610 665 emission filter, $1 \mathrm{sec}$ of exposure time. The different fluorescence signals (which are due to various undefined chemical compounds) allow to recognise a gradient in the pulp from the more external skin-adjacent zone (blue) throughout the middle part (green) to the most inner one (yellow-red).

\section{CONCEPTUAL BASES}

When the light passes through a biological material, the photons encounter different structures with different optical refractive index, which lead to a change of the photon original direction. This phenom- 
enon is known as scattering (Council, 1996). A photon can undergo multiple scattering events (10-1000 or more), depending on both the photon wavelength and the optical properties of the specimen, before fatally encountering a molecule which provokes the absorption of the energy carried by the photon and its disappearance. Alternatively, the photon can reach the sample surface and can be detected.

If a molecule absorbs a photon, it is excited to a higher vibrational energy level and it rapidly loses part of the energy as heat. Then, the molecule eventually relaxes to the ground state emitting a photon characterized by a lower energy with respect to the first one. This almost immediate process $\left(10^{-8} \mathrm{sec}\right)$ is called fluorescence.

Another mechanism of de-excitation of the molecule is called phosphorescence, and it is related to a forbidden transition between energy levels, the first one with long decay times (Tuchin, 2007).

\section{FLUORESCENCE LUMINESCENCE IMAGING (FLI) TECHNIQUE}

The process of fluorescence is induced by illumination of the sample and, indeed, FLI needs external light sources to excite the subject, and the light detection during illumination. When the excitation source switches-off, the fluorescence emission immediately ends (Tuchin, 2015). In addition to the light source, filters are employed to discriminate the emitted photons (with lower energy) from the exciting photons (with higher energy). Fluorescent items detectable by FLI can be proteins (e.g., the green fuorescent protein, GFP), dyes (Luker and Luker, 2008; Balas, 2009), and nanoparticles (objects with the three dimension smaller than $100 \mathrm{~nm}$, see Boschi and De Sanctis, 2017). FLI allows linking fluorescent dyes or nanoparticles to biomolecules of interests: thus it can be applied for an in vivo evaluation of the biodistribution of marked molecules (often pharmaceuticals) in order to assess their uptake in the different anatomical districts.

It is worth noting that the exposure time for FLI acquisitions is in the order of only one second and many samples can be acquired simultaneously, making FLI investigations very simple and easy.

When investigating autofluorescence, which is due to endogenous fluorophores, the interpretation of the results is not straightforward due to the presence of many different fluorescent molecules in the biological systems (Croce and Bottiroli, 2014). 


\section{BiOLUMiNESCENCE IMAGING (BLI) TECHNIQUE}

The bioluminescence is a form of chemi-luminescence that is the production and emission of light by a living organism. BLI is based on the acquisition of the light emitted by the reaction between the substrate luciferin and the enzyme luciferase. The light emission of this enzyme-substrate reaction has a broad spectrum peaked around 600 $\mathrm{nm}$ (Luker and Luker, 2008) but can vary depending on the luciferase. BLI is especially used for in vivo monitoring of tumour growth or for in vivo (Luker and Luker, 2008) or in vitro reporter studies (Prescher and Contag, 2010).

The monitoring of tumour progression is based on the in vitro transfection of cancer cells for luciferase expression and their inoculation in living system. Once luciferin is intraperitoneally injected in the animal, the light produced by the luciferin-luciferase reaction reaches the maximum 12-15 minute after injection, remaining stable up to 40 minutes. Only living cells expressing luciferase are detected by the OI technique; therefore, since the other cells show a negligible background light signal, the images acquired are characterized by a high signal-tobackground ratio. This approach allows detecting the primary tumour but also metastatic cancer masses spread in the living organism, which are formed by the same cancer cells. Generally the exposure time for BLI acquisitions is of the order of 5 min.

\section{INSTRUMENT}

The instrument for OI acquisition is generally called optical imager and consists of a tight light box to avoid that the measurement could be contaminated from the ambient light. It is also made of a very high sensitive CCD camera ( 512 × 512 up to 2048 × 2048 pixels). The reduction/limitation of the electronic noise in guaranteed by cooling of the CCD between -40 and $-90^{\circ} \mathrm{C}$. The samples are placed in the dark chamber. In the case of small animals, they are placed under anaesthesia inside the instrument on a heated stage (generally $37^{\circ} \mathrm{C}$ ) to preserve the body temperature during image acquisitions.

For FLI, the instruments are equipped with one or more light sources (xenon lamp, lasers or light-emitting diodes - LEDs) to illuminate the samples, and a set of excitation/emission filters are employed 
to select the appropriate excitation light specific for the fluorophores, and to discriminate the right wavelengths reaching the detector.

Since BLI is based on the acquisition of light produced by a luminescent structures in the sample, no source of light is required.

The operator can set a field of view (FoV) from few $\mathrm{cm}$ to 20-30 $\mathrm{cm}$, and select several other parameters, such as the exposure time, diaphragm, binning (i.e. the sensitivity of the CCD) and focus height. The luminescence images acquired are provided in pseudo-colours associated to the intensity of the light of each image pixel. In order to quantify the fluorescence emission, the operator can trace a region of interest (ROI) on the luminescent images of the samples. In FLI, the light efficiency is generally measured, which is calculated by normalizing the fluorescence emission to the incident excitation intensities, whereas in BLI the total flux emitted on the surface of the samples is evaluated.

\section{AN APPLICATION OF OI TO GRAPE WITHERING}

As already mentioned, OI is an interesting technique useful for the analysis of a wide range of samples. We used OI in a pilot study for evaluating berries of Corvinone cultivar during their withering for the production of Amarone wine, to see if an optical imager instrument could highlight changes in fluorescence emission which may actually be related to the modification of the berry during the shrivelling process.

Amarone is a very famous dry red wine, typical of the Veneto region in Italy, whose production is characterized by several steps among which the critical phase of withering (Parronetto and Dellaglio, 2011).

Once harvested, the Corvinone berries were placed in plastic boxes located in the fruttaio, a large loft where a continuous exchange of air is guaranteed. We performed a first collection of berries immediately post-harvest (time-point 0 ), followed by other two samplings, one month (time-point 1) and three months (time-point 2) post harvesting.

For each time-point, we monitored by OI the fluorescence emission of the entire berry in FLI modality. Ten excitation filters (from 430 to $745 \mathrm{~nm}$ ) and 18 emission filters (from 500 to $840 \mathrm{~nm}$ ) for a total of 111 excitation/emission filter combinations were used. An example of fluorescent emission from grapes is reported in Fig. $2 \mathrm{~A}$. The fluorescent excitation/emission pattern of Corvinone cultivar, obtained with the 111 excitation/emission filters is shown for the three time-points (Fig. 2 B). 
A
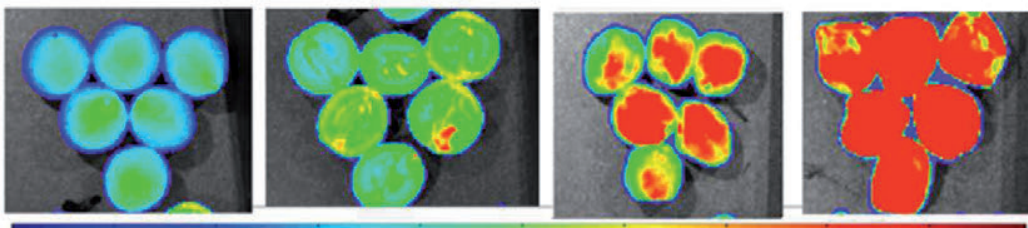

Efficiency 4

6

8

10

12

14

$16 \quad 18$

$20 \times 106$

B
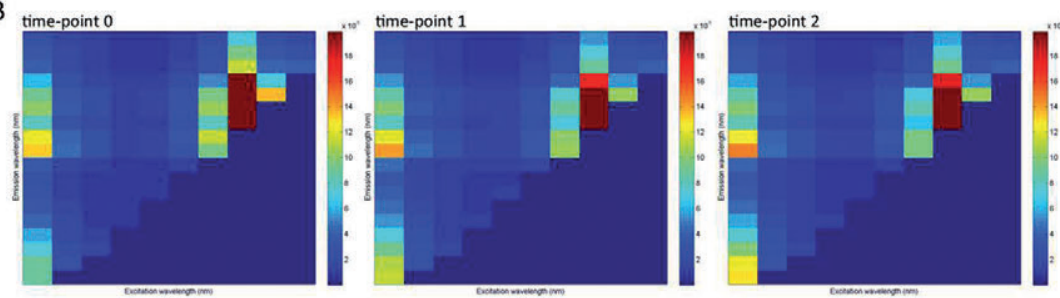

Fig. 2. A) Examples of fluorescence images of Corvinone berries obtained with different combination of excitation and emission filters (430/540, 430/6, 675/720, 710/760 nm excitation/emission filters). The variations in fluorescence emission are dependent on the various molecules excited by the different filter setups; B) Fluorescent excitation/emission diagram of Corvinone cultivar obtained by the fluorescence signal acquired at time-point 0 , time-point 1 and time-point 2.

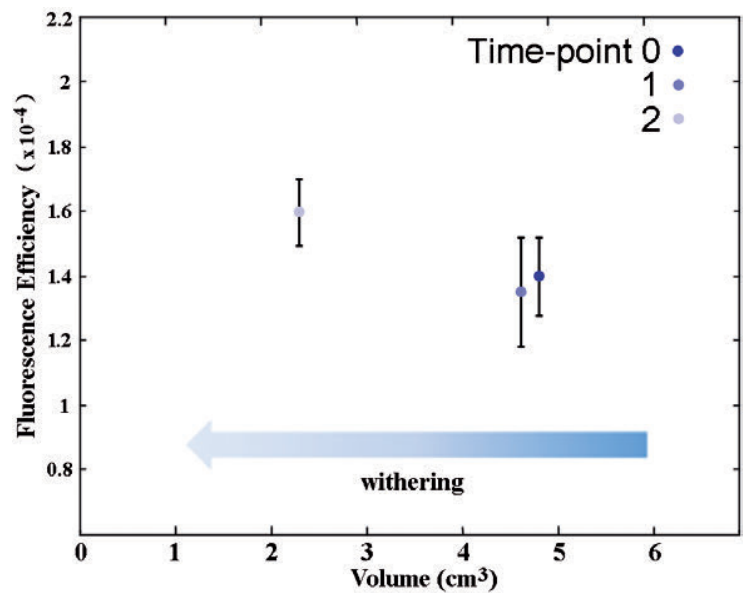

Fig. 3. In the Corvinone berries, the fluorescence efficiency increases concomitantly with the volume reduction due to withering. Measurements were taken with 430/680 excitation/emission filters, i.e. under the conditions which best highlight the pulp fluorescence signal. 
Interestingly, the graph in Fig. 3 shows that, from time-point 0 to time-point 2, the volume of the Corvinone berries decreases in concomitance with an increase of the pulp fluorescence efficiency (Cisterna et al., 2018). This result may be consistent with a concentration of some molecules in the pulp with the withering of the berry leading to an enhancement of their fluorescence signal.

As already mentioned, in order to precisely detect which compound promotes the endogenous fluorescent signal revealed, other imaging and spectroscopic techniques should support OI in a multimodal approach (Cisterna et al., 2018).

\section{REFERENCES}

Balas C. Review of biomedical optical imaging - A powerful, non-invasive, non-ionizing technology for improving in vivo diagnosis. Meas Sci Technol 2009; 20:1-12.

Boschi F, De Sanctis F. Overview of the optical properties of fluorescent nanoparticles for optical imaging. Eur J Histochem 2017, 61:245-248.

Cisterna B, Boschi F, Croce AC, Podda R, Zanzoni S, Degl'innocenti D, et al. Ozone treatment of grapes during withering for Amarone wine: a multimodal imaging and spectroscopic analysis. Microsc Microanal 2018; 24:564-573.

Council NR. Mathematics and Physics of Emerging Biomedical Imaging. Washington DC: The National Academies Press 1996.

Croce AC, Biottiroli G. Autofluorescence Spectroscopy and Imaging: A Tool for Biomedical Research and Diagnosis. Eur J Histochem 2014; 58:2461.

Luker GD, Luker KE. Optical imaging: current applications and future directions. J Nucl Med 2008; 49:1-4.

Paronetto L, Dellaglio F. Amarone: A modern wine coming from an ancient production technology. Adv Food Nutr Res 2011; 63:285-306.

Prescher JA, Contag CH. Guided by the light: visualizing biomolecular processes in living animals with bioluminescence. Curr Opin Chem Biol 2010; 14:80-9.

Tuchin V. Tissue Optics: Light Scattering Methods and Instrument for Medical Diagnosis. Washington DC: SPIE Press 2007.

Tuchin V. Tissue Optics: Light Scattering Methods and Instruments for Medical Diagnosis. Washington DC: SPIE Press 2015. 